Review Article

\title{
Gut Microbiome Dysbiosis and Immunometabolism: New Frontiers for Treatment of Metabolic Diseases
}

\author{
José E. Belizário $\mathbb{D}^{1},{ }^{1}$ Joel Faintuch, ${ }^{2}$ and Miguel Garay-Malpartida ${ }^{3}$ \\ ${ }^{1}$ Department of Pharmacology, Institute of Biomedical Sciences, University of São Paulo, SP CEP 05508-900, Brazil \\ ${ }^{2}$ Department of Gastroenterology of Medical School, University of São Paulo, SP CEP 05403-000, Brazil \\ ${ }^{3}$ School of Arts, Sciences and Humanities (EACH), University of São Paulo, São Paulo, SP CEP 03828-000, Brazil
}

Correspondence should be addressed to José E. Belizário; jebeliza@usp.br

Received 23 February 2018; Accepted 23 October 2018; Published 9 December 2018

Guest Editor: Fabio S. Lira

Copyright ( 2018 José E. Belizário et al. This is an open access article distributed under the Creative Commons Attribution License, which permits unrestricted use, distribution, and reproduction in any medium, provided the original work is properly cited.

\begin{abstract}
Maintenance of healthy human metabolism depends on a symbiotic consortium among bacteria, archaea, viruses, fungi, and host eukaryotic cells throughout the human gastrointestinal tract. Microbial communities provide the enzymatic machinery and the metabolic pathways that contribute to food digestion, xenobiotic metabolism, and production of a variety of bioactive molecules. These include vitamins, amino acids, short-chain fatty acids (SCFAs), and metabolites, which are essential for the interconnected pathways of glycolysis, the tricarboxylic acid/Krebs cycle, oxidative phosphorylation (OXPHOS), and amino acid and fatty acid metabolism. Recent studies have been elucidating how nutrients that fuel the metabolic processes impact on the ways immune cells, in particular, macrophages, respond to different stimuli under physiological and pathological conditions and become activated and acquire a specialized function. The two major inflammatory phenotypes of macrophages are controlled through differential consumption of glucose, glutamine, and oxygen. M1 phenotype is triggered by polarization signal from bacterial lipopolysaccharide (LPS) and Th1 proinflammatory cytokines such as interferon- $\gamma$, TNF- $\alpha$, and IL- $1 \beta$, or both, whereas M2 phenotype is triggered by Th2 cytokines such as interleukin-4 and interleukin-13 as well as anti-inflammatory cytokines, IL-10 and TGF $\beta$, or glucocorticoids. Glucose utilization and production of chemical mediators including ATP, reactive oxygen species (ROS), nitric oxide (NO), and NADPH support effector activities of M1 macrophages. Dysbiosis is an imbalance of commensal and pathogenic bacteria and the production of microbial antigens and metabolites. It is now known that the gut microbiota-derived products induce low-grade inflammatory activation of tissue-resident macrophages and contribute to metabolic and degenerative diseases, including diabetes, obesity, metabolic syndrome, and cancer. Here, we update the potential interplay of host gut microbiome dysbiosis and metabolic diseases. We also summarize on advances on fecal therapy, probiotics, prebiotics, symbiotics, and nutrients and small molecule inhibitors of metabolic pathway enzymes as prophylactic and therapeutic agents for metabolic diseases.
\end{abstract}

\section{Introduction}

Human microbiomes refer to collective genomes of bacteria, archaea, viruses, protozoans, and fungi that coinhabit multiple ecosystems in the human body (Bäckhed et al. [1], Belizario and Napolitano [2]). An adult man of $70 \mathrm{~kg}$ might contain up to $3.8 \times 10^{13}$ bacteria, which is equal to the number of cells of an adult human body (Sender et al. [3]). Bacteria are morphologically and biochemically classified based on various properties including wall type, shape, requirement of oxygen (anaerobic or aerobic), endospore production, motility, and their metabolism. Bacteria are also classified by phylogenetic diversity of variable nucleotide sequences of small subunit ribosomal RNA operons or $16 \mathrm{~S}$ and $18 \mathrm{~S}$ rRNA genes $[1,2]$.

The Human Microbiome Project (HMP) have been defining criteria for high-quality and comprehensive metagenomic analysis of genetic material recovered directly from distinct sites on the human body to determine the microbial relative abundance of multiple strains and species of different phyla at physiological conditions [4-6]. Advances on computational techniques have allowed studies of public 
human metagenomes based on the phylogenetic clustering and assembly of bacterial genomes into taxonomic domain, kingdom, phylum, class, order, family, genus, and species [4-8]. The analyses of various human microbiome datasets revealed the immense diversity at both populations and individuals over evolutionary and lifetime [7-9]. The gut microbiota of healthy individuals is composed of permanent and transitory microbial species and subspecies of over 17 candidate bacterial phyla belonging to Firmicutes $(>70 \%)$, Bacteroidetes $(>30 \%)$, Proteobacteria $(<5 \%)$, Actinobacteria $(<2 \%)$, Fusobacteria and Verrucomicrobia $(<1 \%)$, and other phyla. The novel bacterial genome assembly and taxonomic profiling based on 1550 metagenome-assembled genomes (MAGs) have revealed nearly 70,000 bacterial and archaeal genomes and new species that are under deep investigation [6-8]. A common set of prevalent microbial species found in normal human stools includes the Clostridiales species such as Coprococcus, Ruminococcus, Eubacterium, Bacteroides dorei and fragilis, and Alistipes finegoldii and onderdonkii $[7,8]$. The fully broad taxonomic distribution, microbial evolution, and metabolism of bacterial species regarding caloric load and nutrient absorption have served to cluster species-level phylotypes into major human enterotypes [9-12]. The prevalent human enterotype type 1 is characterized by high levels of Bacteroides and type 2 by few Bacteroides but high levels of Prevotella. They are, respectively, associated with individuals that ingest either high content of animal protein (type 1) or carbohydrates (type 2).

The gastrointestinal (GI) tract possesses its own nervous system known as the enteric nervous system. This system communicates with the central nervous system through nerves, such as the vagus, neuromodulators, and neurotransmitters of sympathetic and parasympathetic branches of the autonomic nervous system [1]. Bacterial richness and diversity in the GI microbiota occupy a central role in normal metabolic and immunological functions of tissues and organs $[1,2]$. Here, we will update on diverse studies exploring the roles of microbiomes, nutrients, and metabolites on immune cell function, etiology of inflammatory, and metabolic diseases, including their wide range of mechanisms. We also describe why and how novel dietary and pharmacological strategies including fecal transplantation, probiotics, prebiotics, and small molecules may help to treat and modulate species-level phyla types and promote the restoration of microbiomes causing metabolic syndromes.

\section{Gut Microbiota Controls the Host's Metabolic Physiological States}

Microorganisms in the gut perform their functions largely through enzyme pathways, in order to digest complex dietary carbohydrates and proteins $[13,14]$. Gut microbiota provides the branched-chain amino acids leucine, isoleucine, and valine, and particularly glycine, which is required for the synthesis of glutathione-the main intracellular antioxidant and detoxifying agent necessary for many biological functions of the host. Bacteria of the gut synthesize a large variety of signaling molecules of low molecular weight that include methane, hydrogen sulfide, and nongaseous metabolites
$[13,14]$. Those products are able turn on or off both host genes and microbe virulence and metabolism genes. Microorganisms also sense diverse environmental signals, including host hormones and nutrients, and respond to them by differential gene regulation and niche adaptation $[13,14]$.

The maintenance of a stable, fermentative gut microbiota requires diets rich in whole plant foods, particularly rich in fibers $[13,14]$. These substrates are processed by the intestinal microbiota enzymes, such as glycoside hydrolases and polysaccharide lyases to produce polyamines, polyphenols, and vitamins $\mathrm{B}$ and $\mathrm{K}$. Under anaerobic conditions, species belonging to the Bacteroides genus, and to the Clostridiaceae and Lactobacillaceae families, in special, Citrobacter and Serratia strains, produce short-chain fatty acids (SCFAs) which are volatile fat acids able to cross the blood-brain barrier via monocarboxylate transporters. SCFAs produced by intestinal bacteria are acetate ( 2 carbon atoms), propionate (3 carbon atoms), and butyrate ( 4 carbon atoms), and their molar ratios vary from $3: 1: 1$ to $10: 2: 1$, respectively. Most of SCFAs are metabolized to $\mathrm{CO}_{2}$. Butyrate acts on colonocytes, goblet cells, and Paneth cells and provides energy for cellular metabolism and regulates apoptosis, cellular differentiation, and chemical modification of nuclear proteins and nucleic acid. Acetate and propionate pass into the bloodstream and are taken up by the liver and peripheral organs, where they can act as substrates for gluconeogenesis and lipogenesis $[14,15]$. The G-protein-coupled receptors (GPRs), GPR41 and GPR43, also named free fatty acid receptors 2 and 3 (FFARs 2/3) present in many tissues, including adipose, gut enteroendocrine cells, and inflammatory cells act as major receptors of SCFAs [14, 15]. Under certain physiological conditions, SCFAs can induce the secretion of glucagon-like peptides (GLP-1 and GLP-2) and peptide YY (PYY). GLP1 stimulates $\beta$ cells of the pancreas to produce insulin, whereas PYY inhibits nutrient absorption in the intestinal lumen as well as control the appetite. The gut microbiota contributes to fat deposition through the regulation of the nuclear farnesoid $\mathrm{X}$ receptor (FXR), the bile acid receptor that is responsible for the regulation of bile acid synthesis, and hepatic triglyceride accumulation [16]. Bile acids, for example, deoxycholic acid, have antimicrobial effects on microbes of the gut and also induce the synthesis of antimicrobial peptides by gut epithelial tissue [17]. Moreover, microbiota converts carnitin and choline to trimethylamine and thus regulates directly the bioavailability of choline and indirectly the accumulation of triglycerides in the liver. The gut microbiota also helps the absorption of calcium, magnesium, and iron. High or low productions of SCFAs, tryptophan metabolites, GABA, noradrenaline, dopamine, acetylcholine, and 5-hydroxytryptamine (serotonin) are associated with various inflammatory and metabolic diseases and neuropsychiatric disorders [18]. Some of these factors act as major neurotransmitters and modulators of the brain-gut axis, and serotonin has central roles in sexuality, substance addiction, appetite, emotions, and stress response [18].

Thousands of microbiota-derived metabolites with known and unknown functions have been identified as components of the human metabolome [19-21]. GI microbiota produces 
large quantities of epigenetically active metabolites, such as folate and $A$ and $B$ vitamins (including riboflavin $\left(B_{2}\right)$, niacin $\left(B_{3}\right)$, pantothenic acid $\left(B_{5}\right)$, pyridoxine $\left(B_{6}\right)$, folate $\left(B_{9}\right)$, and cobalamin $\left(B_{12}\right)$ ) that regulate the activity of host chromatin-modulating enzymes and genetic responses to environmental signals [22]. Acetyl-CoA produced by a number of metabolic processes is the acetyl donor for histone modification (acetylation and deacetylation) catalyzed by histone acetyltransferases. Glycine, serine, and methionine are substrates for DNA methylation and demethylation enzymes. Therefore, changes in gut microbiota can result in epigenomic changes not only directly in adjacent intestinal cells but also in distant cell lineages, such as hepatocytes and adipocytes [22]. Finally, bacteria can inhibit the growth of their competitors by long distance microbial communication, via release of metabolites and quorum sensing peptides, which are considered a biological strategy for maintenance of density of commensal species, and elimination of pathogenic bacteria $[23,24]$.

\section{Gut Microbiota Drives Host Immunological Functions}

A collection of $100-400 \times 10^{12}$ of bacteria inhabits and lives in mutualistic relationships in the human GI tract [3]. The GI double mucus layer is formed by heavily O-glycosylated mucin proteins encoded by MUC2 gene of the mucin protein family. Most of the bacteria of the colon are tightly attached to the outer mucus layer, and the inner layer forms a physical barrier that limits bacterial contact with the epithelium. Most of microbial species in GI tract are transmitted at early life to babies through mother's milk, which contains predominantly Bifidobacteria and Lactobacillus species [25, 26]. Along the transition of infancy to adult life, increase in food sources drives the complexity and diversity of bacterial communities of the genera Bacteroides, Parabacteroides (Bacteroidetes), and Clostridium (Firmicutes) [1, 2, 25, 26]. Bacterial density in the jejunum/ileum $\left(<10^{5}\right)$ and in the large intestine progressively increases in comparison with the stomach and duodenum, and the highest taxa and cell density are present in the colon, which contains $10^{9}-10^{12}$ colony-forming units per $\mathrm{ml}$ (99\% of total GI population). They are anaerobes such Bacteroides, Porphyromonas, Bifidobacterium, Lactobacillus, and Clostridium. Anaerobic bacteria and oxygen-sensitive microbes are capable of producing of short-chain fatty acids than facultative aerobic bacteria such as E. coli, by a factor of 1000. Disruption of the dynamic interrelation between the host and the microbial communities causes dysbiosis, which is a bacterial imbalance between aerobic and facultative anaerobic bacteria ratios $[1,2]$. Hypoxia prevents the growth of pathogenic facultative anaerobes such as $E$. coli and Salmonella. The rupture of gut barrier provoked by dysbiosis leads to local and systemic inflammation [1, 2, 27]. A study by Byndloss et al. unequivocally demonstrated that dysbiosis could be caused by high levels of oxygen and nitrates, which are compounds that contribute to the growth of Escherichia and Salmonella species [28]. Many diseases, including inflammatory bowel diseases (IBDs), irritable bowel syndrome (IBS), diabetes, obesity, and cancer, have been associated with specific bacterial dysbiosis [29-31]. The potential temporary shifts of microbiota species, for example, by the reduction of anti-inflammatory species, such as Faecalibacterium prausnitzii and Akkermansia muciniphila which predominate in healthy individuals, or by the proliferation of potentially proinflammatory bacteria such as Bacteroides and Ruminococcus gnavus, can promote the disease progression and chronicity [29-32]. Comparative studies in lean and obese animal models have indicated that low Bacteroidetes/Firmicutes ratio is a hallmark of obesity [33]. Firmicutes rely heavily on dietary carbohydrates, whereas Proteobacteria rely on proteins as carbon source. For instance, Akkermansia muciniphila is a mucindegrading bacterium in the phylum of Verrucomicrobia that is present in great abundance in healthy humans, but is present in reduced number in patients with inflammatory and gastrointestinal diseases, obesity, and type 2 diabetes (T2D) [34]. In fact, distinct microbial communities and mechanisms can drive host's susceptibility to diseases and influence on clinical outcomes [35].

The gut microbiota plays a critical role in the immune system by controlling the development and functionality of gut-associated lymphoid tissues (GALT), including Peyer's patches, isolated lymphoid follicles, and mesenteric lymph nodes [30, 31, 36, 37]. Microbes and their products are necessary for the immune system to distinguish self from nonself (invaders) at early life and activation and maintenance of innate hematolymphoid cells (ILC1, 2, and 3), natural killer (NK) cells, and cytotoxic and noncytotoxic and helper lymphoid cells [36-39]. NK cells and ILC1 produce large amounts of IFN- $\gamma$, antimicrobial peptides (AMPs), granulysin, defensins, lysozyme, and Reg III $\gamma$, which together play critical functions on the regulation of microbial ecology and immune surveillance $[24,31,39,40]$. For instance, polysaccharide A, $\alpha$-galactosylceramide, and tryptophan metabolites produce by microbial communities stimulate immune cells to produce interleukin-22, Reg3 $\gamma$, IgA, and interleukin-17 [37]. IgA is one important component of innate response to prevent invasion of the microorganisms into circulation [30]. T helper (Th) 17 cells and regulatory $\mathrm{T}$ cells (Treg) are antigen-specific populations that respond to transforming growth factor- $\beta$ and retinoic acid and control immune tolerance $[38,39]$. This control of whole body immune system by gut bacteria appears to be a delicate framework since loss of a specific species can lead to overreaction or suppression of the innate immune response $[27,35,36]$.

A variety of membrane and intracellular receptors named "pattern recognition receptors" or PRRs expressed on the epithelial and immune cells act as sensors of bacterial and cellular products, which are named the pathogen-associated molecular patterns (PAMPs) and damaged-associated molecular patterns (DAMPs) $[41,42]$. PAMPs and DAMPs such as lipopolysaccharides (LPS), lipid A, peptidoglycans, flagellin, microbial RNA/DNA, as well as host cell constituents, such as uric acid, HMGB1 (high-mobility group box 1 protein), double-stranded DNA, and mitochondrial, are recognized by the members of the Toll-like receptor (TLR) family and nuclear oligomerization domain-like receptor of 


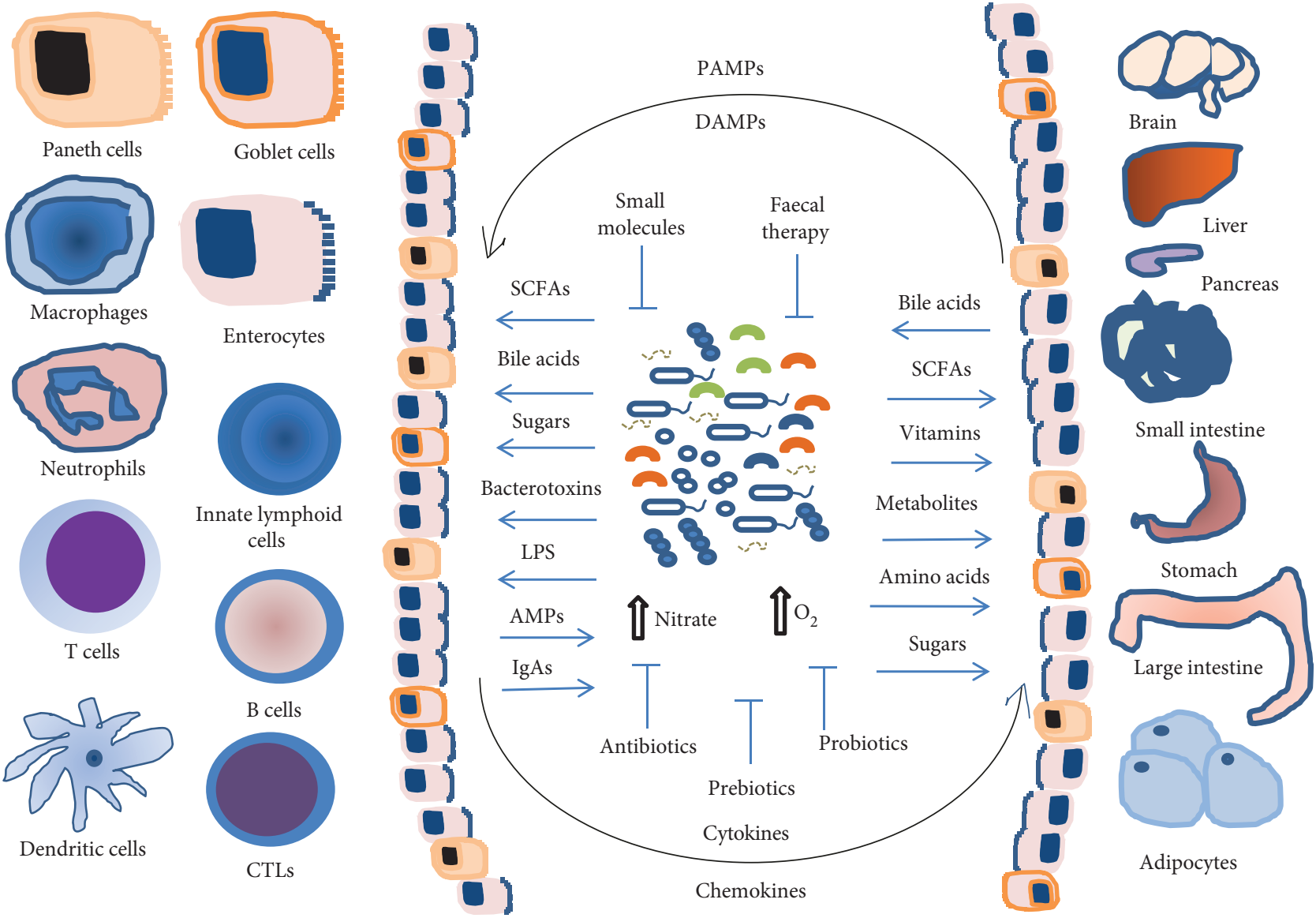

FIGURE 1: Interplay of gut microbiome-intestinal epithelial cells (enterocytes, goblet cells and paneth cells) and host metabolism and immunity. The commensal, symbiotic, and pathogenic microorganisms provide a great variety of nutrients and metabolites for host metabolism, for energy homeostasis of organs and tissues, and for the innate and adaptive immune cell activation and function. A shift toward dysbiosis results from a decrease in symbiont and/or an increase in pathobiont bacteria in intestinal lumen. Increases in nitrate and oxygen $\left(\mathrm{O}_{2}\right)$ allow the growth of facultative anaerobic bacteria. Increase in the gut permeability and release of PAMPs, such as peptidoglycan and LPS, and DAMPs, such as double-stranded RNA, mtDNA, and ATP. Increase in the production of cytokines, chemokines, nitric oxide (NO), and reactive oxygen species (ROS) by dendritic cells and macrophages causes local and systemic inflammation. Chronic, low-grade systemic inflammation leads to impaired insulin action, insulin resistance, obesity, hypertension, and metabolic syndrome. Probiotics, prebiotics, fecal therapy, and small molecules targeting host genes and specific bacterial species or phylum/class may help to reestablish tissue homeostasis and microbiome healthy. SCFAs: short-chain fatty acids; PAMPs: pathogen-associated molecular patterns; DAMPs: damage-associated molecular patterns; LPS: lipopolysaccharide; AMPs: antimicrobial peptides; IgA: immunoglobulin A.

NOD/NLR family $[41,42]$. Extracellular and intracellular complexes formed by DAMPs and PAMPs and NOD/ NLR receptors constitute the inflammasomes [43]. These cytosolic complexes associate with the adapter protein ASC (apoptosis-associated speck-like protein) and proinflammatory proteases of caspase family caspase 1 , caspase 11 , caspase 4 , and caspase 5 [43]. Following the inflammasome activation occurs the production of interleukins IL- $1 \beta$ and IL-18. These cytokines increase the synthesis of other cytokines such as TNF- $\alpha$, IL-6, IL-17, IL-22, and IL-23 and several active chemical inflammatory mediators [43].

Various studies using gene-deficient mice models have defined the direct and complex interplay of bacterial dysbiosis and genetic and environmental factors [35, 36, 44]. Many inflammatory diseases are caused by mutations or loss of some innate response genes in lymphoid tissues and smaller Peyer's patches and mesenteric lymph nodes $[38,39]$. The components of inflammasomes such as
MyD88, TLRs, NODs, NLrp3/6, ASC, and caspase 1 and caspase 11 are known to play a control of the intestinal dysbiosis $[29,38,39,45,46]$. For instance, $\mathrm{NOD}^{-} I^{-}$and NOD2 ${ }^{-} I^{-}$ transgenic mice have increased susceptibility not only to inflammatory bowel disease but also to type 1 diabetes and cancer $[38,39,45,46]$. The animal housing conditions and diet-induced microbiota composition are some examples that may be responsible for strain phenotypic differences in transgenic animals [47, 48]. Germ-free (GF) mice display underdeveloped lymphoid tissues, impairment of $\mathrm{T}$ and $\mathrm{B}$ cell function, and decreased $\mathrm{CD} 4^{+} \mathrm{T}$ cells and antibody production. Their Th17 and Treg cells are less efficient in the control of infection. Colonization of GF mice with limited number of bacterial species (gnotobiotic mouse models) can restore immunological functions $[47,48]$. The phenotypes observed in these mice models are not always observed in human studies. It is important to mention that $85 \%$ of the murine microbiome species have not been detected in human 
microbiomes [49, 50]. Furthermore, the humanization of mouse models with human cells or human microbiota cannot adequately display the whole spectrum of relevant human disease phenotypes $[47,48]$.

Figure 1 displays major classes of molecules, metabolites, and nutrients produced by bacterial species, immune cells, tissues, and organs that regulate the dynamic interplay between host cells and gut microbiome, as well as therapeutic strategies for controlling dysbiosis and diseases.

\section{Immunometabolism and Mitochondrion Reprogramming Pathways}

Mitochondria serve as the powerhouse of the cell by producing and releasing critical signals to the environment and synthesizing ATP, the body's energy required for metabolic processes [51]. The bioenergetic pathways of glycolysis, the tricarboxylic acid (TCA) cycle (also known as Krebs cycle and the citric acid cycle), and fatty acid and amino acid metabolism are central metabolic processes for complete oxidation of all nutrients in the mitochondria $[51,52]$. Cells use aerobic glycolysis to produce glucose-derived pyruvate that is converted into acetyl coenzyme A (acetyl-CoA). Acetyl-CoA molecules derived from glucose, glutamine, or fatty acid metabolism enter in the TCA cycle and are converted into $\mathrm{CO}_{2}, \mathrm{NADH}$, and $\mathrm{FADH}_{2}$ during oxidative phosphorylation (OXPHOS) OXPHOS occurs through passing electrons along a series of carrier molecules, called the electron transport chain, with the help of electron carriers, such as $\mathrm{NAD}(\mathrm{P}) \mathrm{H}$ and $\mathrm{FADH}_{2}$, that serve as substrate to generate adenosine triphosphate (ATP) in the mitochondrial matrix [51, 52]. The electrons are transferred from $\mathrm{NADH}$ to $\mathrm{O}_{2}$ through three protein complexes: NADH dehydrogenase, cytochrome reductase, and cytochrome oxidase. Electron transport between the complexes occurs through other mobile electron carriers, ubiquinone and cytochrome c. The newly synthesized ATP is transported to the cytosol by adenine nucleotide translocase in exchange for ADP. Acetyl-CoA is the precursor for the synthesis of cholesterol and fatty acids, which are incorporated in the cellular plasma membranes.

Immunometabolism comprehends the hub of biochemical activities carry out by immune cells to modulate gene expression profile and switching metabolic pathways and their key enzymes [53]. After a stimulatory signal, various immune cells, in particular, macrophages, DCs, and T cells, exhibit distinct reprogramming metabolic pathways to promote their activation, survival, and lineage generation [53]. This reprogramming in the metabolic pathways is best characterized in macrophages and is illustrated in Figure 2. A seminal study by Newsholme et al. led to the discovery that the consumption rate of glucose, glutamine, and fatty acids and the enzymatic activities of glucose-6-phosphate dehydrogenase, 6-phosphogluconate dehydrogenase, citrate synthase, oxoglutarate dehydrogenase, and glutaminase differ in resting and elicited (inflammatory) macrophages [54]. Their study demonstrated that all glucose utilized by inflammatory macrophages was converted into lactate and very little of it was oxidized $[54,55]$. Since then, the shift from oxidative phosphorylation (OXPHOS) toward glycolysis and glutaminolysis has been considered as a metabolic reprogramming pathway of the inflammatory cells. Remarkably, macrophages, $\mathrm{T}$ cells, and among other immune cells use glycolysis for rapid production of radical oxygen species (ROS) used for limiting infection. Glycolysis also provides rapid production of ATP and metabolic intermediates for the synthesis of ribose for nucleotides and amino acids for the biosynthesis by RNA and DNA of proteins by macrophages. Glycolysis is a preferential pathway for the activation of dendritic cells, $\mathrm{CD}^{+} \mathrm{T}$ helper 1 (Th1), Th2, and Th17 cells, NK cells, and cytotoxic $\mathrm{CD} 8^{+}$cells $[51,52]$. Interesting, this metabolic adaptation to aerobic glycolysis (in the presence of oxygen) was first described as a hallmark of tumor cells by Warburg et al. in a seminal report published 60 years ago [56].

Unstimulated macrophages displaying M0 phenotype and exhibiting the differentiation surface markers $\mathrm{CD}^{+} 8^{+} /$ CD80/low $/ C D 206^{\text {high }}$ acquire and display the M1 phenotype $\left(\mathrm{CD} 68^{+} / \mathrm{CD} 80 /^{\text {low }} / \mathrm{CD} 206^{\text {low }}\right)$ or $\mathrm{M} 2$ phenotype $\left(\mathrm{CD}^{+} 8^{+} /\right.$ $\mathrm{CD} 80^{\text {high }} / \mathrm{CD} 206 /{ }^{\text {low }}$ ) after switching their metabolism and function in response to stimuli or polarization signals that initiate a pro- or anti-inflammatory response [57]. M1 cells display Th1-oriented proinflammatory effector properties and promote tissue damage and antimicrobial and antitumor resistance, whereas M2 cells exhibit tissue remodeling and repair functions, promote wound healing, angiogenesis, and resistance to parasites, and favor tumor growth. The reprogramming metabolic pathways in M1/M2 macrophages alter their functions, including cytokine production, phagocytosis, and antigen presentation [58-60]. The classical activation pathway or reprogramming pathway in M1 macrophages promotes the accumulation of citrate and high production of NO, ROS, cytokines, and prostaglandins [60,61]. M1 macrophages release ROS and NO in phagosomes where they promote the killing of pathogens. Th2 cytokines, such as IL-4 and IL-13, regulate macrophage alternative pathway or M2 reprogramming pathway. The major feature of M2 reprograming pathways is that TCA cycle occurs coupled to oxidative phosphorylation, $\beta$-oxidation of fatty acids, and mitochondrial biogenesis. In addition, M2 macrophages do not produce NO. It is important to consider that M2 macrophages display various distinct forms (M2a, b, and c) depending on the local tissue environment and exposure to stimuli [57].

The generation of UDP-GlcNAc intermediates promotes the glycosylation of M2-associated receptors, such as the mannose receptor [60]. Thus, both macrophage M1 and M2 phenotypes can be controlled by either oxygen and nutrients or cytokines and damage- and pathogen-associated molecular pattern- (DAMP- and PAMP-) mediated signals [60]. These events are controlled by the signaling and transcriptional pathways induced by canonical regulators of cellular metabolism, such as C-myc transcription factor, coactivator proteins such as PPAR $\gamma$ and PGC- $1 \beta$ (peroxisome proliferative-activated receptor $\gamma$, coactivator $1 \beta$ ), and signaling pathways driven by AMPK ( $5^{\prime}$-adenosine monophosphate-activated protein kinase), mTORC1/ 2 (mammalian target of rapamycin complexes), and STAT6 


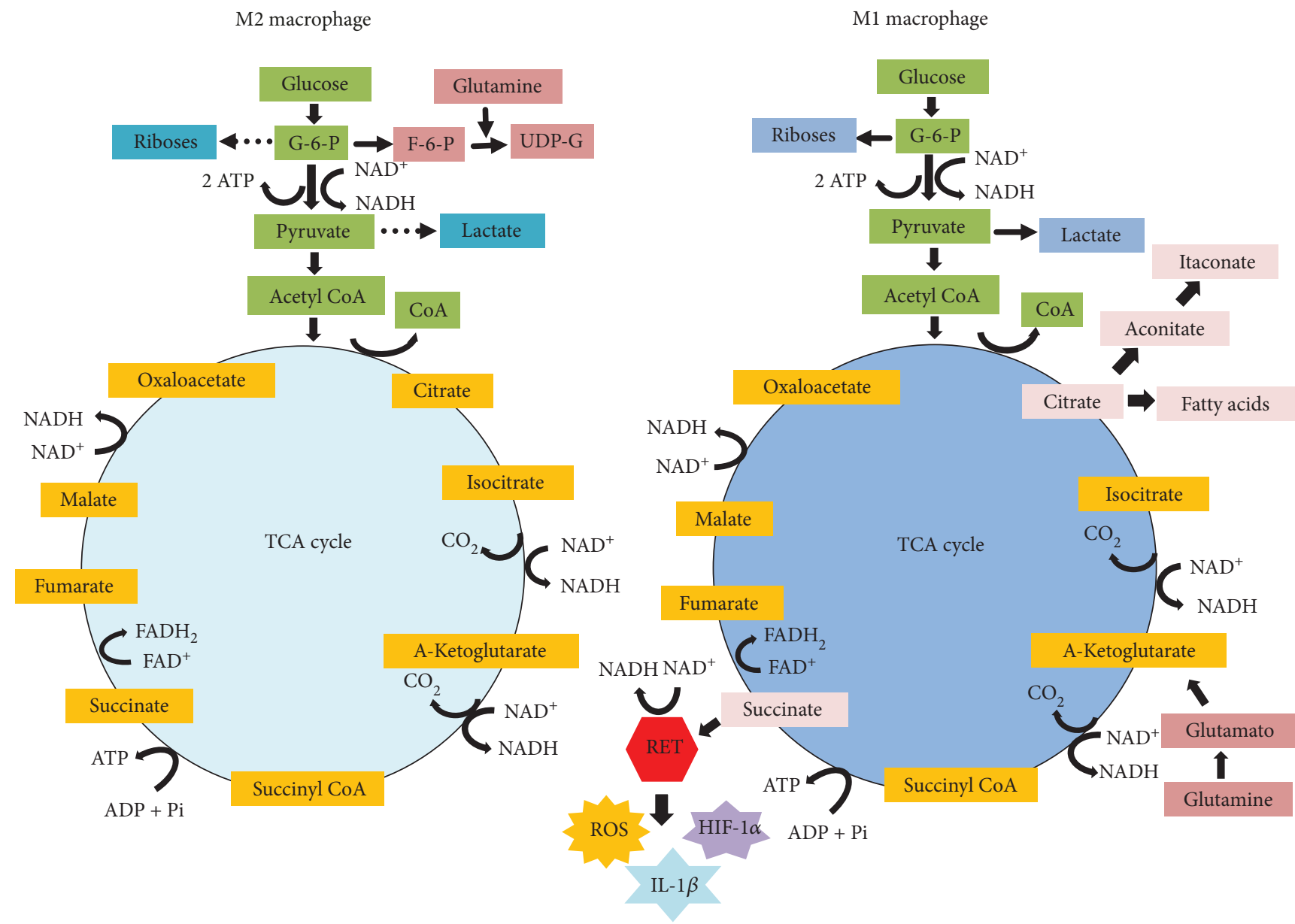

FIGURE 2: Metabolic pathways supporting macrophage reprogramming in either M2 or M1 phenotype. IL-4-stimulated M2 macrophage utilizes glycolysis, TCA, and mitochondrial oxidative phosphorylation (OXPHOS) to generate NADPH and ATP for energy. G-6-P and glutamine are used for generation of UDP-Glc-Nac required for receptors and protein glycosylation. LPS-stimulated M1 macrophage produces G-6-P that is reduced to pyruvate, in parallel, $\mathrm{NAD}^{+}$is reduced to NADPH, and 2 ATP molecules are produced. In hypoxia, pyruvate is reduced to lactate, restoring $\mathrm{NAD}^{+}$to glycolysis recycle, and thus, OXPHOX is uncoupled to glycolysis. TCA cycle in LPS-stimulated M1 macrophageis deviated after citrate and after succinate. Citrate is used for the syntheses of NO, ROS, and prostaglandins. Citrate is used by the enzyme immune-responsive gene 1 (IRG1) to generate itaconate that acts as an endogenous SDH inhibitor and also as antimicrobial. Succinate is oxidized by SDH and subsequently used for production of ROS from a complex 1 via reverse electron transport (RET). Succinate activates HIF- $1 \alpha$ which in turn increases the transcription of IL- $1 \beta$. Glutaminolysis provides NADPH for generation of ROS. SDH: succinate dehydrogenase; UDP-Glc-Nac: uridine diphosphate N-acetylglucosamine; NO: nitric oxide; ROS: reactive oxygen species. Arrows indicated the direction of reaction and products.

(signal transducer and activator of transcription) [51, 52]. A deep overview of macrophage metabolic pathways and phenotypic and functional outcomes is described elsewhere [62].

In 2013, Tannahil et al. reported that expression of IL-1 $\beta$ mRNA in M1 macrophages stimulated with LPS leads to activation of the transcription factor hypoxia-induced factor $1 \alpha(\mathrm{HIF} 1 \alpha)$, in a glycolysis-dependent manner [63]. HIF $1 \alpha$ interacts with pyruvate kinase isoenzyme M2 (PKM2) and promotes the expression of HIF $1 \alpha$-induced genes required for glycolysis [64]. The activation of the Warburg effect (glycolysis) in LPS-stimulated macrophages causes an accumulation of intermediates of the TCA cycle, in particular, succinate, malate, and fumarate due to a flux deviation or a "break point" of the Krebs cycle pathway (see Figure 2). Succinate exerts multiple immunological functions $[52,53$, 63]. Oxidation of succinate by the enzyme succinate dehydrogenase (SHD), which converts succinate to fumarate, drives the production of ROS from complex II in the mitochondrion, a process named reverse electron transport (RET). Macrophages respond to activation of HIF- $1 \alpha$ via ROS and increasing the expression of IL- $1 \beta$ [58]. Inhibition of SDH with dimethylmalonate inhibits IL-1 $\beta$ expression, while increasing the production of immunosuppressive cytokine IL-10 [52, 63].

Itaconate is one important metabolite formed in the second flux deviation or 'break point' of the Krebs cycle pathway during macrophage transition from inactive to proinflammatory state [65]. Itaconate is an unsaturated dicarboxylic acid produced by extra mitochondrial enzyme cys-aconitate decarboxylase encoded by immune-responsive gene 1 (Irg1). This enzyme converts cys-aconitate (derived from citrate) to itaconic acid. One of remarkable effect 
observed after itaconate treatment is the reduction of the expression of cytokines IL-1 $\beta$, IL-6, and iNOS [65]. Studies using murine bone marrow-derived macrophages (BMDMs) and RAW-264.7 cells, stimulated with LPS and cytokines, showed that the absence (knockdown) of Irg gene leads to impairment in substrate-level phosphorylation (SLP) of mitochondria [66]. SLP is a metabolic reaction that results in the formation of ATP or GTP by the direct transfer of phosphate group to ADP or GDP from another phosphorylated compound. Administration of itaconate (0.5-2 mM) reverses this reaction [66]. Studies using transgenic mice and immune cells deficient of Irg 1 gene demonstrated that itaconate acts as an endogenous succinate dehydrogenase inhibitor and that such inhibition causes the accumulation of succinate [67]. Together, these studies confirmed that itaconate regulates succinate levels, mitochondrial respiration, and inflammatory cytokine production and, therefore, acts as an important regulator of macrophage activation. Furthermore, itaconic acid has antimicrobial activity and kills directly intracellular Salmonella typhimurium, Legionella pneumophila, and Mycobacterium tuberculosis; thus, it protects against infection. The cytotoxic effect is associated with the inhibition of the enzyme isocitrate lyase [68]. Isocitrate lyase regulates a bacterial specific metabolic pathway known as the glyoxylate shunt, in which acetyl-CoA is converted to succinate for the synthesis of carbohydrates. In this pathway, isocitrate is cleaved by the enzyme ICL (encoded by aceA) yielding glyoxylate and succinate, which reenter into TCA cycle following the oxidative decarboxylation steps. Thus, the glyoxylate shunt acts as microbial survival pathway [66-68].

\section{Gut Dysbiosis Associated with Metabolic Diseases}

Studies on the relationship among gut microbes, obesity, insulin resistance, and metabolic syndrome have shown an intricate interplay between host diet, genetics, and microbiome compositional dynamics [49, 69, 70]. A series of experiments have shown that a chronic inflammatory process through translocation of gut bacterial LPS into the bloodstream initiates a silent metabolic endotoxemia and ultimately obesity-related disorders [71-73]. The hallmarks of clinical manifestations of metabolic syndrome include central obesity, high blood pressure, and high levels of blood sugar and serum triglycerides, which are most significant drifts to the development of insulin resistance, type 2 diabetes, hypertension, and fatty liver disease. Individuals robustly colonized by bacteria and archeae of the genera Faecalibacterium, Bifidobacterium, Lactobacillus, Coprococcus, and Methanobrevibacter have significantly less tendency to develop metabolic disturbances and inflammation and, in turn, type 2 diabetes and ischemic cardiovascular disorders $[49,73]$. These species are higher producers of SCFAs and hydrogen peroxides, which are compounds known to inhibit biofilm formation by pathogenic species, including Staphylococcus aureus and E. coli [73].

Obesity is associated with behavioral and environmental factors, such as excessive consumption of energy-dense foods and sedentary lifestyle [49, 73-75]. Initial clues for the role of microorganisms in energy homeostasis and obesity appeared from the studies using germ-free animals [76]. Several important questions on the complex interaction between host and microbes in pathophysiology of obesity remain answered [49, 74, 75]. Studies in genetic and diet-induced mouse models of obesity confirmed that the ratio of Firmicutes to Bacteroidetes is increased in obese animals, as compared to nonobese control animals [33, 49, 77]. The higher ratio of Firmicutes to Bacteroidetes was also found in clinical studies that evaluated overweight and obese healthy volunteers [33], in high total amount of fecal SCFAs was detected [78]. Mouse and human models have demonstrated inverse relationships between A. muciniphila colonization and inflammatory conditions. In fact, A. muciniphila is present in low levels in people suffering from morbid obesity, diabetes, and cardiometabolic diseases supporting their role as antiobesity strain [79].

The contribution of GI hypothalamic-pituitary-adrenal axis for predisposition to obesity is still incompletely understood $[18,70]$. Recently, one study described the relationship of high-fat diet and the levels of acetate produced by intestinal microbiota in a rat model [80]. The authors concluded that chronic acetate turnover activates the parasympathetic nervous system, which coordinates the secretion of glucosestimulated insulin, ghrelin, and hyperphagia. They conclude that together these factors cooperate in the promotion of obesity [80]. High-fat diet-induced obesity leads also to chronic low-grade hypothalamic inflammation and activation of both microglia and astrocytes [81]. A study using mice model suggests that acetate accumulated in the hypothalamus. This compound plays a central role in prevention of weight gain through an anorectic effect [82]. The neuroimmuno-endocrine pathway is crucial for glucose homeostasis and control of adiposity in obese people recovering from bariatric surgery [80]. After bariatric surgery by Roux-en-Y gastric bypass (RYGB) methods, human and animal models change food preference $[83,84]$. Normally, a fatty acid derivative named oleoylethanolamide (OEA) is produced after ingesting a fat meal. OEA can directly activate PPAR$\alpha$ receptors, which are responsible for promoting satiety via vagus nerve and dopamine release in the brain [84]. Dopamine-suppressed obese animals display low levels of OEA in the brain. After RYGB surgery, these animals increase the levels of OEA and dopamine 1 receptor (D1R) expression, which promote a shift in GI-brain axis signaling. This leads to dramatic change in animal feeding behavior which includes the preference for low-fat food [84]. RYGB is also known to profoundly affect the secretion of many gastrointestinal hormones, including ghrelin and GLP-1 [83]. Administration of exogenous GLP-1 or GLP-1 analog promotes weight loss and glucose regulation in T2DM patients. Remarkably, metformin, a drug used to treat T2DM, can increase butyrate-producing bacteria and thereby promote the restoration of the healthy microbiome [85]. Together, these discoveries have opened new perspectives for future therapies to improve health by targeting pivotal host cells, microbes, and microbiome-derived metabolites. 


\section{Targeting Gut Microbiota Dysbiosis and Metabolic Pathways}

6.1. Probiotics and Prebiotics. The immunologist Elie Metchnikoff was the first to defend that the ingestion of fermented milk (i.e., yogurt) prepared with Bacillus bulgaricus increases health and prolong life span. He anticipated the rational use of probiotic made of live microorganisms with a health benefit through altering the gut microbiome [86]. Lactobacillus plantaram and Bifidobacterium are probiotic bacteria capable of modulating negative effects of high-fat diets and even managing immunological reactions mediated by inflammatory diseases $[25,38,45]$. Bifidobacterium and Lactobacillus are producers of folate in the gut. Lactobacillus rhamnosus in combination with Lactobacillus gasseri and Bifidobacterium lactis may reduce weight gain, in particular, fat tissue mass adiposity, in humans [87]. Studies on the effects of $A$. muciniphila alive or pasteurized, in high-fat diet-fed mice, revealed that a small $30 \mathrm{kDa}$ Amuc_1100 protein activates TLR2, allowing the development of an intestinal health immune response [79].

Dietary prebiotics are known to provide immune and metabolic benefits to the host $[14,86]$. The poorly digestible carbohydrates, such as nonstarch polysaccharides, resistant starch, nondigestible oligosaccharides (NDOs), and polyphenols, are source of various sugars including glucose, galactose, rhamnose, and rutinose. The carbohydratehydrolyzing enzymes of colonic microbiota promote the fermentation of prebiotics, and these produce hydrogen, methane, carbon dioxide, and SCFAs. When associated, the probiotic and prebiotic can selectively stimulate growth and activity of health-promoting bacteria $[14,86]$. In this way, probiotic and prebiotics have potential to modulate metabolic processes involved in T2DM and obesity-related disorders $[14,86]$. Ingestion of inulin or oligofructose improves metabolic disorders associated with obesity, including insulin resistance and metabolic endotoxemia [34, 71, 79]. These effects may be associated with restoration of gut barrier integrity and reduction of LPS release from Gram-negative bacteria $[25,88]$.

6.2. Fecal Microbial Transplant (FMT). The use of antibiotics permanently modifies gut microbial community [89]. High doses and frequency of antibiotics, particularly against anaerobes, such as vancomycin, can disrupt and destabilize normal gut microbiome. Extended spectrum antibiotic causes the overgrowth of Clostridium difficile and chronic recurrent colitis [89]. Fecal bacteriotherapy is a clinical procedure, in which a liquid suspension of stool from a human donor (a family member or a disease-free screened donor) is inoculated into gut's patients to restore gut microbiota in refractory cases of Clostridium difficile colorectal infection after antibiotic therapy $[90,91]$. These fecal preparations may contain from $3.0 \%$ to $10 \%$ of viable, dead bacteria and colonic cells and other components that impact on transplant outcomes, by enhancing or inhibiting immune function of innate lymphoid cells (ILCs) [92]. Today, more than 150 clinical trials involving FMT are being conducted, mostly in the USA to treat a large array of metabolic, infectious, and immunological diseases and complications, including kidney- and liver-transplanted patients, especially with antibiotic-resistant bacterial colonization or infection (ClinicalTrials.gov).

Studies have shown that around $90 \%$ of the patients that received FMT for Clostridium difficile infection were cured, as compared to $31 \%$ of those receiving only vancomycin (van Nood et al. [93]). Lactobacillus, Enterococcus, Bifidobacterium, and Bacteroides are known to exert an inhibitory activity against $C$. difficile growth. For instance, a variety of viruses, archaea, fungi, parasitic species, and metabolites are transferred together with FMT [92]. Thus, further studies are needed to confirm their ability to potentially promote host intestinal immunity while optimizing microbiome diversity.

A series of animal model studies have shown that the gut microbiome plays a crucial role in weight gain and obesity $[49,50,70]$. Studies on lean and genetically obese (ob/ob) mice and rats have revealed great difference in their intestinal absorption and microbiome composition, which are related to $50 \%$ reduction in Bacteroidetes and proportional increases in Firmicutes and Archaea species [74, 75]. Transferring the microbiota from fat mice to germ-free mice hosts induces greater weight gain than in those receiving the microbiota from lean donors $[49,50]$. High-fat intake increases intestinal permeability and diffusion of LPS associated with obesity. In contrast, the administration of Bifidobaterium infantis in mice reduces the production of proinflammatory cytokines while promoting white adipose tissue gain $[68,71]$. Results of FMT treatment of people with diverse metabolic disorders have not been conclusive. Transfer of intestinal microbiota from lean donors mildly increased insulin sensitivity in subjects with metabolic syndrome [94]. Short exposure to antibiotics may improve peripheral insulin sensitivity in a small number of obese subjects [94]. Furthermore, in a study with 75 obese and prediabetic volunteers that underwent an 8-day antibiotic treatment (amoxicillin and vancomycin), the antibiotic-driven dysbiosis did not alter gut permeability, as confirmed by variation in LPS levels and expression of lipid metabolic enzymes [95]. Such heterogeneity may reflect complex interactions between genetic, lifestyle, and environmental factors derived from different model systems.

6.3. Small Molecules. Acetate, propionate, and butyrate are the most important SCFAs that eventually are given as oral dietary supplementation. SCFAs acting as signaling molecules produce various physiological effects in humans [96]. They bind to GPR41 and GPR43 receptors (free fatty acid receptors 2 and 3, FFARs 2/3) in intestinal L cells signaling the release of GLP-1 and peptide YY [78]. Notably, injection of GLP-1 peptides stimulates insulin signaling in white adipose tissues, therefore reducing adiposity. SCFAs also increase leptin secretion and adipogenesis while inhibiting lipolysis of adipose tissues. In the liver, propionate acts as a gluconeogenic factor while acetate and butyrate act as lipogenic factors [96]. SCFAs, particularly, propionic and butyric acids, may directly prevent low-grade inflammatory response in obesity by controlling gut microbiota [88]. Butyrate acting on human monocyte, marrow-derived DCs, and 
macrophages inhibits IL-12 production, decreases costimulatory molecule expression, and blocks NF- $\kappa \mathrm{B}$ translocation [37]. Butyrate and structural analog and ketone body $\beta$ hydroxybutyrate (also known as 3-hydroxybutyrate) supplementation can inhibit the activity of histone deacetylases (HDACs). HDACS promote specific histone modifications to regulate transcription and DNA replication and repair. It also exerts anti-inflammatory activity by suppressing NF- $\kappa$ B and STAT1 activation [22-95]. Succinate is a well-known inhibitor of the histone demethylases (DNMTs) and the eleven translocation (TET) methylcytosine dioxygenases, which oxidize 5-methylcytosines to promote DNA demethylation [22].

Several small molecule inhibitors of metabolic pathways such as 2-deoxy-glucose, dichroacetate, BPTES (bis-2-(5-phenylacetamido-1,3,4-thiadiazol-2-yl)ethyl sulfide), dimethylmalonate (DMM), rotenone, and metformin have identified as promise therapeutics [51, 52, 60]. Metformin is a firstline medication for the treatment of T2D that significantly improves metabolic parameters such as body weight, insulin, glucose, leptin, and C-reactive protein plasma levels [96]. By inhibiting complex I in mitochondrial respiration chain, metformin elevates the plasma levels of lactate. Lactic acidosis is a critical problem in clinical practice. Metformin causes a switch from OXPHOS to aerobic glycolysis [97]. Thus, users of metformin have a higher risk of metforminassociated lactic acidosis. Metformin treatment increases significantly the relative abundance of $A$. muciniphila in the fecal microbiota in obese mice [34]. A. muciniphila is a producer of acetate and propionate, through mucin degradation, and T2D patients treated with metformin increase their butyrate- and propionate-producing bacteria, which may in turn contribute to the beneficial effect of metformin [85]. It is interesting to ask if metformin effects are also associated with M1/M2 macrophage metabolic reprogramming pathways.

Succinate is a strong proinflammatory mediator [51, 63]. DMM inhibits mitochondrial ROS production through inhibition of succinate dehydrogenase (SDA), which converts succinate to fumarate. This metabolic alteration limits the production IL- $1 \beta$, while increasing the synthesis of immunosuppressive IL-10 [67]. TEPP-46 and DASA-58, two small molecule inhibitors of PKM2 (pyruvate kinase isozyme 2), inhibit LPS-induced HIF- $1 \alpha$ and IL- $1 \beta$, thereby promoting the reprogramming of M1 macrophages to M2 $[63,98]$. Thus, further studies are required to confirm these pharmacological targets and approaches to control metabolic disorders.

\section{Conclusions and Perspectives}

Over the last decade, the sequencing and analyses of a large number of human microbiomes and assembly of their metabolic pathways have expanded our understanding of how bacterial metabolites participate in the microbe-host interactions in health and diseases. The functional variations of gut microbiomes among individuals have indicated the anabolic and catabolic pathways of essential importance in maintaining core community structures for whole body homeostasis. Emerging new biomarkers are promising specific discrimination of phyla and species to confirm evidences for direct participation of microbes in type II diabetes, obesity, metabolic disorders, inflammatory bowel diseases, and even certain cancers.

There is no doubt that dysbiosis, by altering microbiome metabolism and consequently host metabolism, not only affects inflammatory responses and adaptive immunity but also contributes to metabolic disorders. The use of innovative pharmaceutical and nutraceutical products to manage microbial colonization and development of a healthy gut microbial community at early childhood and adult life may prevent the occurrence of common inflammatory and metabolic pathologies. Finally, the discovery and development of drugs that target enzymes of metabolic pathways, and also drive pro- and anti-inflammatory responses of immune cells, will provide the next frontier medicine for metabolic therapies in near future.

\section{Conflicts of Interest}

The authors declare that they have no conflicts of interests.

\section{Authors' Contributions}

JEB, JF, and MGM conducted the literature review process and selected articles and wrote the manuscript and prepared figures. The authors read and approved the final manuscript.

\section{Acknowledgments}

We thank colleagues of the University of Sao Paulo and Clinical Hospital of Medical School for insights and productive discussions. Financial support from the Coordenação de Aperfeiçoamento de Pessoal de Nível Superior (2011/ 188-37), the Conselho Nacional de Desenvolvimento Científico e Tecnológico (308794/2014-1 and 312206/2016-0), and the Fundação de Amparo à Pesquisa do Estado de São Paulo (2007/04513-1 and 2015/18647-6) is acknowledged.

\section{References}

[1] F. Bäckhed, C. M. Fraser, Y. Ringel et al., "Defining a healthy human gut microbiome: current concepts, future directions, and clinical applications," Cell Host \& Microbe, vol. 12, no. 5, pp. 611-622, 2012.

[2] J. E. Belizario and M. Napolitano, "Human microbiomes and their roles in dysbiosis, common diseases, and novel therapeutic approaches," Frontiers in Microbiology, vol. 6, p. 1050, 2015.

[3] R. Sender, S. Fuchs, and R. Milo, "Revised estimates for the number of human and bacteria cells in the body," PLoS Biology, vol. 14, no. 8, article e1002533, 2016.

[4] J. Qin, R. Li, J. Raes et al., "A human gut microbial gene catalogue established by metagenomic sequencing," Nature, vol. 464, no. 7285, pp. 59-65, 2010.

[5] J. Li, H. Jia, X. Cai et al., "An integrated catalog of reference genes in the human gut microbiome," Nature Biotechnology, vol. 32, no. 8, pp. 834-841, 2014. 
[6] J. Lloyd-Price, G. Abu-Ali, and C. Huttenhower, "The healthy human microbiome," Genome Medicine, vol. 8, no. 1, p. 51, 2016.

[7] J. Lloyd-Price, A. Mahurkar, G. Rahnavard et al., "Strains, functions and dynamics in the expanded Human Microbiome Project," Nature, vol. 550, no. 7674, pp. 61-66, 2017.

[8] D. H. Parks, C. Rinke, M. Chuvochina et al., "Recovery of nearly 8,000 metagenome-assembled genomes substantially expands the tree of life," Nature Microbiology, vol. 2, no. 11, pp. 1533-1542, 2017.

[9] M. Arumugam, J. Raes, E. Pelletier et al., "Enterotypes of the human gut microbiome," Nature, vol. 473, no. 7346, pp. 174-180, 2011.

[10] O. Koren, D. Knights, A. Gonzalez et al., "A guide to enterotypes across the human body: meta-analysis of microbial community structures in human microbiome datasets," PLoS Computational Biology, vol. 9, no. 1, article e1002863, 2013.

[11] E. Le Chatelier, T. Nielsen, J. Qin et al., "Richness of human gut microbiome correlates with metabolic markers," Nature, vol. 500, no. 7464, pp. 541-546, 2013.

[12] A. H. Moeller, Y. Li, E. Mpoudi Ngole et al., "Rapid changes in the gut microbiome during human evolution," Proceedings of the National Academy of Sciences of the United States of America, vol. 111, no. 46, pp. 16431-16435, 2014.

[13] V. Tremaroli and F. Bäckhed, "Functional interactions between the gut microbiota and host metabolism," Nature, vol. 489, no. 7415, pp. 242-249, 2012.

[14] I. Rowland, G. Gibson, A. Heinken et al., "Gut microbiota functions: metabolism of nutrients and other food components," European Journal of Nutrition, vol. 57, no. 1, pp. 1-24, 2018.

[15] K. Meijer, P. de Vos, and M. G. Priebe, "Butyrate and other short-chain fatty acids as modulators of immunity: what relevance for health?," Current Opinion in Clinical Nutrition and Metabolic Care, vol. 13, no. 6, pp. 715721, 2010.

[16] F. J. Gonzalez, C. Jiang, C. Xie, and A. D. Patterson, "Intestinal farnesoid X receptor signaling modulates metabolic disease," Digestive Diseases, vol. 35, no. 3, pp. 178-184, 2017.

[17] J. M. Ridlon, D. J. Kang, P. B. Hylemon, and J. S. Bajaj, "Bile acids and the gut microbiome," Current Opinion in Gastroenterology, vol. 30, no. 3, pp. 332-338, 2014.

[18] S. M. Collins, M. Surette, and P. Bercik, "The interplay between the intestinal microbiota and the brain," Nature Reviews Microbiology, vol. 10, no. 11, pp. 735-742, 2012.

[19] W. R. Wikoff, A. T. Anfora, J. Liu et al., "Metabolomics analysis reveals large effects of gut microflora on mammalian blood metabolites," Proceedings of the National Academy of Sciences of the United States of America, vol. 106, no. 10, pp. 3698-3703, 2009.

[20] N. Pornputtapong, I. Nookaew, and J. Nielsen, "Human metabolic atlas: an online resource for human metabolism," Database, vol. 2015, article bav068, 2015.

[21] S. Abubucker, N. Segata, J. Goll et al., "Metabolic reconstruction for metagenomic data and its application to the human microbiome," PLoS Computational Biology, vol. 8, no. 6, article e1002358, 2012.

[22] Y. Qin and P. A. Wade, "Crosstalk between the microbiome and epigenome: messages from bugs," Journal of Biochemistry, vol. 163, no. 2, pp. 105-112, 2018.
[23] M. B. Miller and B. L. Bassler, "Quorum sensing in bacteria," Annual Review of Microbiology, vol. 55, no. 1, pp. 165199, 2001.

[24] M. J. Ostaff, E. F. Stange, and J. Wehkamp, "Antimicrobial peptides and gut microbiota in homeostasis and pathology," EMBO Molecular Medicine, vol. 5, no. 10, pp. 14651483, 2013.

[25] G. Reid, J. A. Younes, H. C. Van der Mei, G. B. Gloor, R. Knight, and H. J. Busscher, "Microbiota restoration: natural and supplemented recovery of human microbial communities," Nature Reviews Microbiology, vol. 9, no. 1, pp. 27-38, 2011.

[26] D. J. Palmer, J. Metcalfe, and S. L. Prescott, "Preventing disease in the 21st century: the importance of maternal and early infant diet and nutrition," Journal of Allergy and Clinical Immunology, vol. 130, no. 3, pp. 733-734, 2012.

[27] C. R. Webb, I. Koboziev, K. L. Furr, and M. B. Grisham, "Protective and pro-inflammatory roles of intestinal bacteria," Pathophysiology, vol. 23, no. 2, pp. 67-80, 2016.

[28] M. X. Byndloss, E. E. Olsan, F. Rivera-Chávez et al., "Microbiota-activated PPAR- $\gamma$ signaling inhibits dysbiotic Enterobacteriaceae expansion," Science, vol. 357, no. 6351, pp. 570$575,2017$.

[29] J. C. Clemente, L. K. Ursell, L. W. Parfrey, and R. Knight, "The impact of the gut microbiota on human health: an integrative view," Cell, vol. 148, no. 6, pp. 1258-1270, 2012.

[30] E. M. Brown, M. Sadarangani, and B. B. Finlay, "The role of the immune system in governing host-microbe interactions in the intestine," Nature Immunology, vol. 14, no. 7, pp. 660-667, 2013.

[31] C. A. Thaiss, N. Zmora, M. Levy, and E. Elinav, "The microbiome and innate immunity," Nature, vol. 535, no. 7610, pp. 65-74, 2016.

[32] A. Swidsinski, V. Loening-Baucke, H. Lochs, and L. P. Hale, "Spatial organization of bacterial flora in normal and inflamed intestine: a fluorescence in situ hybridization study in mice," World Journal of Gastroenterology, vol. 11, no. 8, pp. 11311140, 2005.

[33] F. J. Verdam, S. Fuentes, C. de Jonge et al., "Human intestinal microbiota composition is associated with local and systemic inflammation in obesity," Obesity, vol. 21, no. 12, pp. E607E615, 2013.

[34] A. Everard, C. Belzer, L. Geurts et al., "Cross-talk between Akkermansia muciniphila and intestinal epithelium controls diet-induced obesity," Proceedings of the National Academy of Sciences of the United States of America, vol. 110, no. 22, pp. 9066-9071, 2013.

[35] U. Roy, E. J. C. Gálvez, A. Iljazovic et al., "Distinct microbial communities trigger colitis development upon intestinal barrier damage via innate or adaptive immune cells," Cell Reports, vol. 21, no. 4, pp. 994-1008, 2017.

[36] J. L. Round and S. K. Mazmanian, "The gut microbiota shapes intestinal immune responses during health and disease," Nature Reviews Immunology, vol. 9, no. 5, pp. 313323, 2009.

[37] A. J. McDermott and G. B. Huffnagle, "The microbiome and regulation of mucosal immunity," Immunology, vol. 142, no. 1, pp. 24-31, 2014.

[38] L. V. Hooper, D. R. Littman, and A. J. Macpherson, "Interactions between the microbiota and the immune system," Science, vol. 336, no. 6086, pp. 1268-1273, 2012. 
[39] K. Honda and D. R. Littman, "The microbiota in adaptive immune homeostasis and disease," Nature, vol. 535, no. 7610, pp. 75-84, 2016.

[40] A. Fuchs and M. Colonna, "Natural killer (NK) and NKlike cells at mucosal epithelia: mediators of anti-microbial defense and maintenance of tissue integrity," European Journal of Microbiology and Immunology, vol. 1, no. 4, pp. 257-266, 2011.

[41] H. Kono and K. L. Rock, "How dying cells alert the immune system to danger," Nature Reviews Immunology, vol. 8, no. 4, pp. 279-289, 2008.

[42] B. Sangiuliano, N. M. Pérez, D. F. Moreira, and J. E. Belizário, "Cell death-associated molecular-pattern molecules: inflammatory signaling and control," Mediators of Inflammation, vol. 2014, Article ID 821043, 14 pages, 2014.

[43] V. A. K. Rathinam and K. A. Fitzgerald, "Inflammasome complexes: emerging mechanisms and effector functions," Cell, vol. 165, no. 4, pp. 792-800, 2016.

[44] M. Mamantopoulos, F. Ronchi, F. van Hauwermeiren et al., "Nlrp6- and ASC-dependent inflammasomes do not shape the commensal gut microbiota composition," Immunity, vol. 47, no. 2, pp. 339-348.e4, 2017.

[45] C. L. Maynard, C. O. Elson, R. D. Hatton, and C. T. Weaver, "Reciprocal interactions of the intestinal microbiota and immune system," Nature, vol. 489, no. 7415, pp. 231241, 2012.

[46] F. R. C. Costa, M. C. S. Françozo, G. G. de Oliveira et al., "Gut microbiota translocation to the pancreatic lymph nodes triggers NOD2 activation and contributes to T1D onset," Journal of Experimental Medicine, vol. 213, no. 7, pp. 1223-1239, 2016.

[47] D. Laukens, B. M. Brinkman, J. Raes, M. De Vos, and P. Vandenabeele, "Heterogeneity of the gut microbiome in mice: guidelines for optimizing experimental design," FEMS Microbiology Review, vol. 40, no. 1, pp. 117-132, 2016.

[48] J. V. Fritz, M. S. Desai, P. Shah, J. G. Schneider, and P. Wilmes, "From meta-omics to causality: experimental models for human microbiome research," Microbiome, vol. 1, no. 1, p. 14, 2013.

[49] R. E. Ley, P. J. Turnbaugh, S. Klein, and J. I. Gordon, "Microbial ecology: human gut microbes associated with obesity," Nature, vol. 444, no. 7122, pp. 1022-1023, 2006.

[50] R. E. Ley, M. Hamady, C. Lozupone et al., "Evolution of mammals and their gut microbes," Science, vol. 320, no. 5883, pp. 1647-1651, 2008.

[51] E. L. Mills, B. Kelly, and L. A. J. O'Neill, "Mitochondria are the powerhouses of immunity," Nature Immunology, vol. 18, no. 5, pp. 488-498, 2017.

[52] K. Ganeshan and A. Chawla, "Metabolic regulation of immune responses," Annual Review of Immunology, vol. 32, no. 1, pp. 609-634, 2014.

[53] L. A. J. O'Neill, R. J. Kishton, and J. Rathmell, "A guide to immunometabolism for immunologists," Nature Reviews Immunology, vol. 16, no. 9, pp. 553-565, 2016.

[54] P. Newsholme, R. Curi, S. Gordon, and E. A. Newsholme, "Metabolism of glucose, glutamine, long-chain fatty acids and ketone bodies by murine macrophages," Biochemical Journal, vol. 239, no. 1, pp. 121-125, 1986.

[55] C. Murphy and P. Newsholme, "Importance of glutamine metabolism in murine macrophages and human monocytes to L-arginine biosynthesis and rates of nitrite or urea production," Clinical Science, vol. 95, no. 4, pp. 397-407, 1998.
[56] O. Warburg, K. Gawehn, and A. W. Geissler, "Metabolism of leukocytes," Zeitschrift für Naturforschung B, vol. 13B, pp. 515-516, 1958.

[57] A. Sica and A. Mantovani, "Macrophage plasticity and polarization: in vivo veritas," The Journal of Clinical Investigation, vol. 122, no. 3, pp. 787-795, 2012.

[58] J. Jantsch, D. Chakravortty, N. Turza et al., "Hypoxia and hypoxia-inducible factor- $1 \alpha$ modulate lipopolysaccharideinduced dendritic cell activation and function," The Journal of Immunology, vol. 180, no. 7, pp. 4697-4705, 2008.

[59] E. Lachmandas, L. Boutens, J. M. Ratter et al., "Microbial stimulation of different Toll-like receptor signalling pathways induces diverse metabolic programmes in human monocytes," Nature Microbiology, vol. 2, no. 3, article 16246, 2017.

[60] L. A. J. O'Neill and E. J. Pearce, "Immunometabolism governs dendritic cell and macrophage function," Journal of Experimental Medicine, vol. 213, no. 1, pp. 15-23, 2016.

[61] V. Infantino, P. Convertini, L. Cucci et al., "The mitochondrial citrate carrier: a new player in inflammation," Biochemical Journal, vol. 438, no. 3, pp. 433-436, 2011.

[62] R. Curi, R. de Siqueira Mendes, L. A. de Campos Crispin, G. D. Norata, S. C. Sampaio, and P. Newsholme, "A past and present overview of macrophage metabolism and functional outcomes," Clinical Science, vol. 131, no. 12, pp. 1329-1342, 2017.

[63] G. M. Tannahill, A. M. Curtis, J. Adamik et al., "Succinate is an inflammatory signal that induces IL- $1 \beta$ through HIF- $1 \alpha$," Nature, vol. 496, no. 7444, pp. 238-242, 2013.

[64] E. M. Palsson-McDermott, A. M. Curtis, G. Goel et al., "Pyruvate kinase M2 regulates Hif- $1 \alpha$ activity and IL- $1 \beta$ induction and is a critical determinant of the Warburg effect in LPS-activated macrophages," Cell Metabolism, vol. 21, no. 1, pp. 65-80, 2015.

[65] V. Lampropoulou, A. Sergushichev, M. Bambouskova et al., "Itaconate links inhibition of succinate dehydrogenase with macrophage metabolic remodeling and regulation of inflammation," Cell Metabolism, vol. 24, no. 1, pp. 158-166, 2016.

[66] B. Németh, J. Doczi, D. Csete et al., "Abolition of mitochondrial substrate-level phosphorylation by itaconic acid produced by LPS-induced Irg1 expression in cells of murine macrophage lineage," The FASEB Journal, vol. 30, no. 1, pp. 286-300, 2016.

[67] T. Cordes, M. Wallace, A. Michelucci et al., "Immunoresponsive gene 1 and itaconate inhibit succinate dehydrogenase to modulate intracellular succinate levels," Journal of Biological Chemistry, vol. 291, no. 27, pp. 14274-14284, 2016.

[68] A. Michelucci, T. Cordes, J. Ghelfi et al., "Immune-responsive gene 1 protein links metabolism to immunity by catalyzing itaconic acid production," Proceedings of the National Academy of Sciences of the United States of America, vol. 110, no. 19, pp. 7820-7825, 2013.

[69] D. Zeevi, T. Korem, N. Zmora et al., "Personalized nutrition by prediction of glycemic responses," Cell, vol. 163, no. 5, pp. 1079-1094, 2015.

[70] I. T. W. Harley and C. L. Karp, "Obesity and the gut microbiome: striving for causality," Molecular Metabolism, vol. 1, no. 1-2, pp. 21-31, 2012.

[71] P. D. Cani, A. M. Neyrinck, F. Fava et al., "Selective increases of bifidobacteria in gut microflora improve high-fat-dietinduced diabetes in mice through a mechanism associated with endotoxaemia," Diabetologia, vol. 50, no. 11, pp. 23742383, 2007. 
[72] R. S. Kootte, A. Vrieze, F. Holleman et al., "The therapeutic potential of manipulating gut microbiota in obesity and type 2 diabetes mellitus," Diabetes, Obesity and Metabolism, vol. 14, no. 2, pp. 112-120, 2012.

[73] T. Arora and F. Backhed, "The gut microbiota and metabolic disease: current understanding and future perspectives," Journal of Internal Medicine, vol. 280, no. 4, pp. 339349, 2016.

[74] P. J. Turnbaugh, R. E. Ley, M. A. Mahowald, V. Magrini, E. R. Mardis, and J. I. Gordon, "An obesity-associated gut microbiome with increased capacity for energy harvest," Nature, vol. 444, no. 7122, pp. 1027-1031, 2006.

[75] P. J. Turnbaugh, M. Hamady, T. Yatsunenko et al., "A core gut microbiome in obese and lean twins," Nature, vol. 457, no. 7228, pp. 480-484, 2008.

[76] F. Backhed, H. Ding, T. Wang et al., "The gut microbiota as an environmental factor that regulates fat storage," Proceedings of the National Academy of Sciences of the United States of America, vol. 101, no. 44, pp. 15718-15723, 2004.

[77] C. De Filippo, D. Cavalieri, M. Di Paola et al., "Impact of diet in shaping gut microbiota revealed by a comparative study in children from Europe and rural Africa," Proceedings of the National Academy of Sciences of the United States of America, vol. 107, no. 33, pp. 14691-14696, 2010.

[78] A. Schwiertz, D. Taras, K. Schäfer et al., "Microbiota and SCFA in lean and overweight healthy subjects," Obesity, vol. 18, no. 1, pp. 190-195, 2010.

[79] P. D. Cani and W. M. de Vos, "Next-generation beneficial microbes: the case of Akkermansia muciniphila," Frontiers in Microbiology, vol. 8, p. 1765, 2017.

[80] R. J. Perry, L. Peng, N. A. Barry et al., "Acetate mediates a microbiome-brain- $\beta$-cell axis to promote metabolic syndrome," Nature, vol. 534, no. 7606, pp. 213-217, 2016.

[81] J. P. Thaler, C. X. Yi, E. A. Schur et al., "Obesity is associated with hypothalamic injury in rodents and humans," The Journal of Clinical Investigation, vol. 122, no. 1, pp. 153162, 2012.

[82] G. Frost, M. L. Sleeth, M. Sahuri-Arisoylu et al., “The shortchain fatty acid acetate reduces appetite via a central homeostatic mechanism," Nature Communications, vol. 5, no. 1, p. 3611, 2014.

[83] R. J. Seeley, A. P. Chambers, and D. A. Sandoval, "The role of gut adaptation in the potent effects of multiple bariatric surgeries on obesity and diabetes," Cell Metabolism, vol. 21, no. 3, pp. 369-378, 2015.

[84] M. K. Hankir, F. Seyfried, C. A. Hintschich et al., "Gastric bypass surgery recruits a gut PPAR- $\alpha$-striatal D1R pathway to reduce fat appetite in obese rats," Cell Metabolism, vol. 25, no. 2, pp. 335-344, 2017.

[85] K. Forslund, F. Hildebrand, T. Nielsen et al., "Disentangling type 2 diabetes and metformin treatment signatures in the human gut microbiota," Nature, vol. 528, no. 7581, pp. 262266, 2015.

[86] M. Roberfroid, "Prebiotics: the concept revisited," The Journal of Nutrition, vol. 137, no. 3, pp. 830S-837S, 2007.

[87] M. C. Mekkes, T. C. Weenen, R. J. Brummer, and E. Claassen, "The development of probiotic treatment in obesity: a review," Beneficial Microbes, vol. 5, no. 1, pp. 19-28, 2014.

[88] P. D. Cani and N. M. Delzenne, "The gut microbiome as therapeutic target," Pharmacology \& Therapeutics, vol. 130, no. 2, pp. 202-212, 2011.
[89] L. Dethlefsen, S. Huse, M. L. Sogin, and D. A. Relman, “The pervasive effects of an antibiotic on the human gut microbiota, as revealed by deep $16 \mathrm{~S}$ rRNA sequencing," PLoS Biology, vol. 6, no. 11, article e280, 2008.

[90] E. Gough, H. Shaikh, and A. R. Manges, "Systematic review of intestinal microbiota transplantation (fecal bacteriotherapy) for recurrent Clostridium difficile infection," Clinical Infectious Diseases, vol. 53, no. 10, pp. 994-1002, 2011.

[91] M. Rupnik, "Toward a true bacteriotherapy for clostridium difficile infection," The New England Journal of Medicine, vol. 372, no. 16, pp. 1566-1568, 2015.

[92] D. P. Bojanova and S. R. Bordenstein, "Fecal transplants: what is being transferred?," PLoS Biology, vol. 14, no. 7, article e1002503, 2016.

[93] E. van Nood, A. Vrieze, M. Nieuwdorp et al., "Duodenal infusion of donor feces for recurrent Clostridium difficile," The New England Journal of Medicine, vol. 368, no. 5, pp. 407-415, 2013.

[94] A. Vrieze, C. Out, S. Fuentes et al., "Impact of oral vancomycin on gut microbiota, bile acid metabolism, and insulin sensitivity," Journal of Hepatology, vol. 60, no. 4, pp. 824-831, 2014.

[95] D. Reijnders, G. H. Goossens, G. D. A. Hermes et al., "Effects of gut microbiota manipulation by antibiotics on host metabolism in obese humans: a randomized doubleblind placebo-controlled trial," Cell Metabolism, vol. 24, no. 1, pp. 63-74, 2016.

[96] D. J. Morrison and T. Preston, "Formation of short chain fatty acids by the gut microbiota and their impact on human metabolism," Gut Microbes, vol. 7, no. 3, pp. 189-200, 2016.

[97] R. DeFronzo, G. A. Fleming, K. Chen, and T. A. Bicsak, "Metformin-associated lactic acidosis: current perspectives on causes and risk," Metabolism, vol. 65, no. 2, pp. 20 29, 2016.

[98] J. C. Alves-Filho and E. M. Pålsson-McDermott, "Pyruvate kinase M2: a potential target for regulating inflammation," Frontiers in Immunology, vol. 7, p. 145, 2016. 


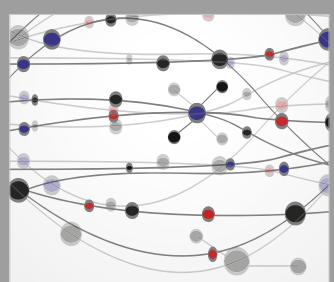

The Scientific World Journal
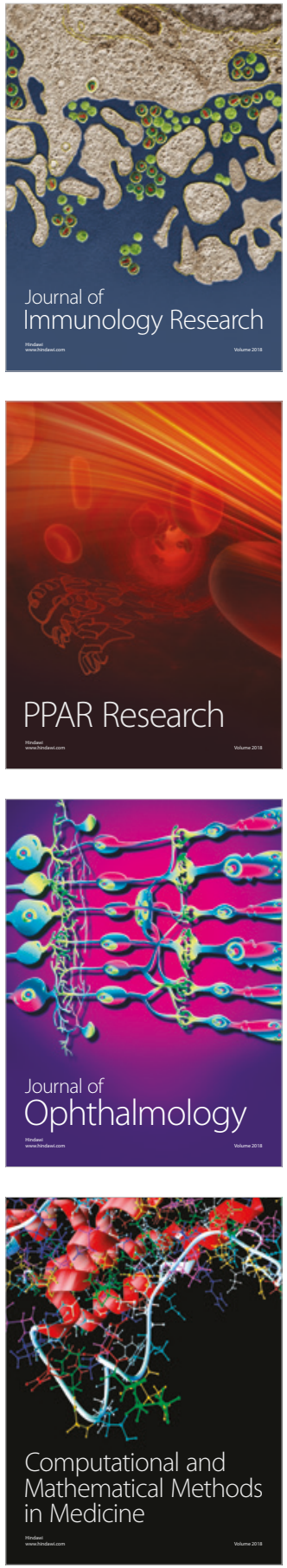

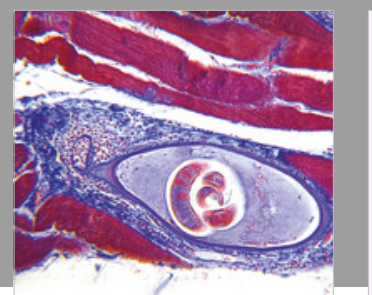

Gastroenterology Research and Practice

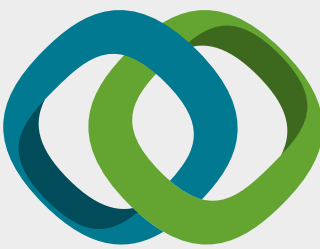

\section{Hindawi}

Submit your manuscripts at

www.hindawi.com
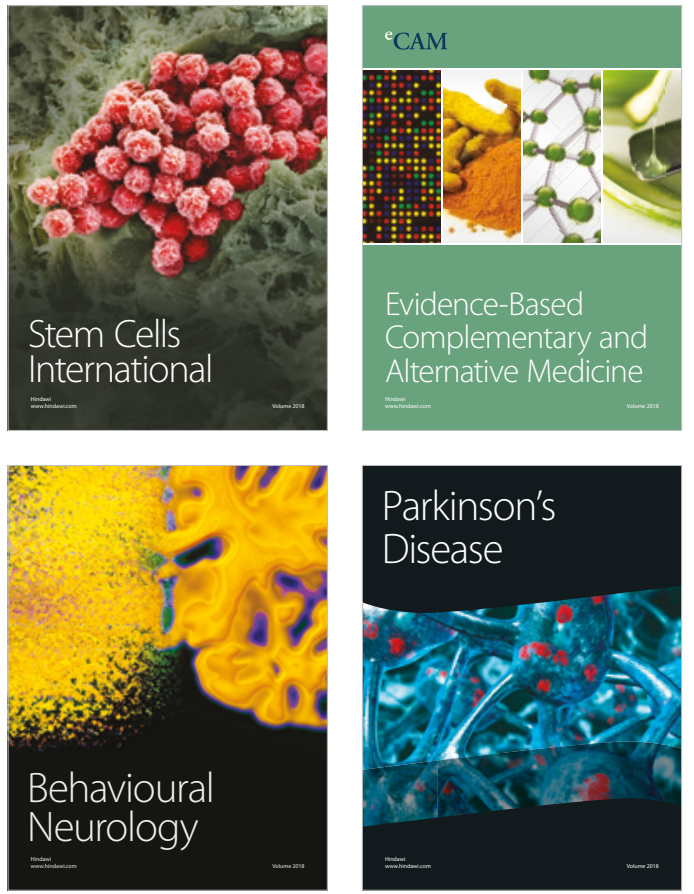

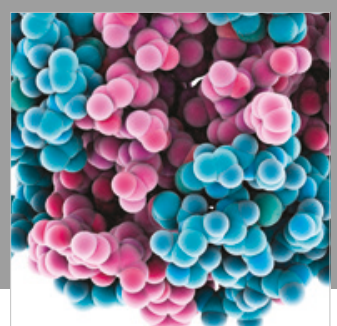

ournal of

Diabetes Research

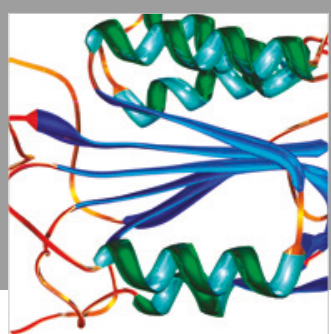

Disease Markers
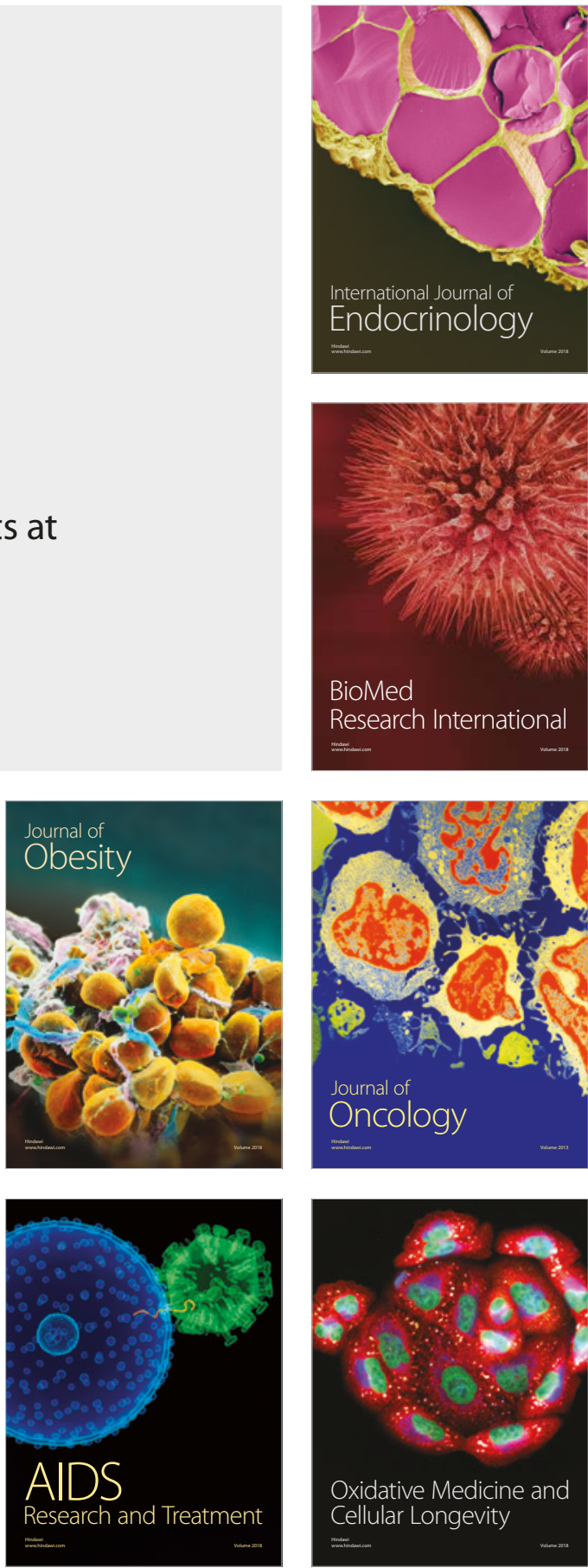